\title{
Achados oculares em pacientes com mais de 99 anos
}

\author{
Ocular findings in patients older than 99 years
}

\author{
Marcela Colussi Cypel ${ }^{1}$ \\ Guilherme Palácio ${ }^{2}$ \\ Paulo Elias Correa Dantas ${ }^{3}$ \\ Cláudio Luis Lottenberg ${ }^{4}$ \\ Rubens Belfort $\mathrm{Jr}^{5}$
}

\section{RESUMO}

Objetivo: Determinar as condições visuais e oculares em indivíduos com mais de 99 anos. Método: Estudo prospectivo e descritivo de série casos. Trinta idosos com mais de 99 anos se inscreveram no Instituto da Visão da Universidade Federal de São Paulo, de forma voluntária, respondendo à busca ativa. $\mathrm{O}$ exame oftalmológico constou de anamnese, ectoscopia, acuidade visual, exame refracional, citologia e cultura de cílios e conjuntiva com antibiograma, teste de Schirmer basal, tempo de ruptura do filme lacrimal, coloração com Rosa Bengala da conjuntiva e córnea, exame de biomicroscopia, tonometria de aplanação e fundoscopia direta e indireta. Foram realizados exames complementares, como retinografia e tomografia de coerência óptica, quando indicados. Resultados: Trinta pacientes acima de 99 anos (média de 101,5 $\pm 1,8$ anos), dos quais, 25 mulheres e 5 homens, foram examinados; destes, 10 não tinham condições de exame completo, em razão de limitações de saúde e mobilidade. A doença sistêmica mais encontrada foi hipertensão arterial $(8$ pacientes $=40 \%)$. Cinco pacientes (25\%) negaram qualquer doença ou uso de medicação. A melhor acuidade visual corrigida para longe foi de 20/100 ou melhor em 11 pacientes $(55 \%)$ e para perto variou de $\mathrm{J} 4$ ou melhor também em 11 pacientes $(55 \%)$, sendo que sete destes idosos (63\%) não haviam sido submetidos à cirurgia de catarata. A principal queixa foi dificuldade para leitura em $55 \%$ e $40 \%$ demonstrou estar satisfeito com a sua visão. A melhora da acuidade com prescrição de óculos novos ocorreu em 4 casos (20\%). Catarata foi identificada como comprometendo a visão de forma significativa em 5 casos (25\%); porém, apenas 2 concordaram com a cirurgia. A principal causa de baixa visão foi a degeneração macular relacionada à idade, presente em todos os casos; na maioria da vezes (95\%), na forma seca. Conclusão: As necessidades da população nessa faixa etária devem ser entendidas para o desenvolvimento de política específica de saúde ocular. Esse estudo demonstrou idosos com maioria do sexo feminino (83,3\%), boa saúde geral (35\%), hipertensos (40\%), com dificuldade para leitura $(55 \%)$ e com baixa visual em decorrência de degeneração macular relacionada à idade.

Descritores: Envelhecimento da população; Envelhecimento/fisiologia; Transtornos da visão; Idoso de 80 anos ou mais; Acuidade visual; Serviços de saúde para idosos; Qualidade de vida

\section{INTRODUÇÃO}

Coletar dados e entender as manifestações das doenças em populações longevas pode ser de grande importância para a saúde pública, em geral, e para a oftalmologia, em particular ${ }^{(1)}$. A importância de pesquisas científicas e estudos epidemiológicos neste grupo etário pode melhorar a terapêutica e 
a prevenção das doenças oftalmológicas diretamente relacionados à qualidade de vida física e psicológica. A população mundial de idosos vem aumentando, permitindo que as doenças próprias dessa faixa etária possam se manifestar e cursar por mais tempo, aumentando a possibilidade de manifestações patogênicas $^{(1-2)}$. Este processo pode ser decorrente: 1) da mudança nos padrões de natalidade (que, com a redução do número de nascimentos, traz um crescimento relativo do número de adultos e idosos); 2) da queda da mortalidade; crescimento absoluto do número de pessoas idosas ${ }^{(3)}$.

O Brasil passou de um perfil de mortalidade típico de uma população jovem com baixos níveis de enfermidades, para um outro perfil, caracterizado por enfermidades complexas e onerosas presentes em populações idosas. Observamos atualmente uma mescla das doenças que os acometem, e que têm características próprias e vêm mostrando uma mudança no seu padrão em decorrência de acometerem por mais tempo esses idosos que tem vivido mais e os avanços da medicina que permitem mudanças na abordagem e acompanhamento destas doenças $^{(4)}$.

O envelhecimento para algumas pessoas é uma fase natural do desenvolvimento e de satisfação, enquanto que para outras é um estádio negativo da vida. A diferença talvez esteja na qualidade de vida, que varia individualmente ${ }^{(5)}$ e na qualidade visual, que é considerada como das mais determinantes, uma vez que a perda visual leva à menor comunicação visual, dependência e restrição de mobilidade, inclusive levando a maior probabilidade de traumas em geral $^{(2,6-7)}$. Os distúrbios visuais, ao promoverem maior dependência física, geram também dificuldades emocionais.

As principais causas de baixa acuidade visual nesta faixa etária são: catarata, alterações na retina e no nervo óptico e glaucoma. Grande parte das mudanças decorrentes do envelhecimento são inevitáveis, mas podem receber cuidados preventivos e curativos, sendo pelo menos postergadas ${ }^{(4-5)}$.

Estimativas mostram que a população mundial acima de 80 anos é de 59,6 milhões sendo destes 29,4 milhões idosos que habitam países menos desenvolvidos (África, América Latina, Oceania). Para 2020, as estimativas são de 101,6 milhões de idosos acima de 80 anos no mundo e 58,2 milhões nos países menos desenvolvidos ${ }^{(8)}$. No Brasil, a expectativa de vida duplicou ao longo do século, em consequiência da queda das taxas de fecundidade e mortalidade ${ }^{(1,3)}$. Considerando idoso aquele com idade igual ou maior a 60 anos, o número registrado de idosos foi de 3 milhões em 1960, passou a 7 milhões em 1975 e atualmente estima-se 15 milhões e a previsão é de que este número poderá dobrar até 2020, chegando a 32 milhões de idosos $^{(3,9)}$. Dados do Instituto Brasileiro de Geografia e Estatística (IBGE) estimam em 2004, 1.939,405 de pessoas acima de 80 anos, destes 1.133,062 são mulheres.

Quanto ao número de centenários no mundo (100 anos ou mais), o número estimado é de 145.000 em 1999 e deverá aumentar em 15 vezes aproximadamente para 2,2 milhões em $2050^{(10)}$. Segundo dados do IBGE, censo de 2000, existem quase 25 mil habitantes com 100 anos ou mais no Brasil.
O objetivo deste estudo foi determinar as alterações oculares presentes em indivíduos com mais de 99 anos.

\section{MÉTODOS}

Trinta idosos com mais de 99 anos procuraram o Serviço de Oftalmologia da Universidade Federal de São Paulo (UNIFESP) e se inscreveram no projeto, de forma voluntária, respondendo à busca ativa a partir de abril de 2004. O exame oftalmológico constou de anamnese, ectoscopia, acuidade visual, exame refracional, citologia e cultura de cílios e conjuntiva com antibiograma, teste de Schirmer basal, tempo de quebra do filme lacrimal, coloração com Rosa Bengala da conjuntiva e córnea, exame de biomicroscopia, tonometria de aplanação e fundoscopia direta e indireta.

Os únicos critérios de exclusão foram: idade inferior a 100 anos e idosos incapacitados de comparecer ao local para o exame.

Dos 30 idosos inscritos 5 eram do sexo masculino e 25 do sexo feminino. Compareceram ao exame 20 idosos, 2 do sexo masculino e 18 do sexo feminino pois os demais encontravam adoentados, hospitalizados ou em casa, sem condição de realizar os exames.

Este trabalho foi aprovado pelo comitê de ética e pesquisa da UNIFESP sob protocolo número 1583/04.

Os exames foram realizados no Instituto da Visão da UNIFESP, sem qualquer custo para o paciente ou fim lucrativo, assim como todo o tratamento clínico e cirúrgico. Os idosos que necessitavam de óculos novos os receberam gratuitamente (doados pela Ótica Miguel Giannini).

Os resultados obtidos foram submetidos à analise estatística descritiva.

\section{RESULTADOS}

A faixa etária foi de 100 anos a 108 anos, com média de idade de 101,5 anos $( \pm 1,8)$.

Em relação às doenças sistêmicas, a mais comum foi hipertensão arterial ( 8 idosos, $40 \%$ ), seguida por artrose (3 idosos, $10 \%$ ), tireoideopatia (1 idoso, $5 \%$ ), asma (1 idoso, $5 \%$ ), cardiopatia ( 1 idoso, $5 \%$ ) e surdez ( 1 idoso, $5 \%)$. Cinco idosos $(25 \%)$ negaram qualquer tipo de doença sistêmica.

Quando questionados sobre a principal queixa visual a resposta encontrada foi de baixa acuidade visual para perto em 11 idosos (55\%). Nove (40\%) mostraram estar satisfeitos com a visão e um idoso por surdez e falta de comunicação, não respondeu a pergunta.

Em 11 idosos (55\%), a melhor acuidade visual para longe (MAVL) foi de 20/100 ou melhor sendo que entre estes 5 (45\%) não tinham sido operado de catarata. Na visão de perto a melhor acuidade visual para perto (MAVP) foi de J4 ou melhor também em 55\% dos casos e destes 7 (63\%) não haviam sido submetidos a cirurgia de catarata (Tabela 1).

$\mathrm{O}$ erro refrativo mais encontrado foi o astigmatismo (10 idosos, $50 \%$ ), sendo cinco com astigmatismo hipermetrópico e 


\begin{tabular}{|c|c|c|c|c|}
\hline \multirow[b]{2}{*}{ Paciente } & \multicolumn{2}{|c|}{ Longe } & \multicolumn{2}{|c|}{ Perto } \\
\hline & OD & $\mathrm{OE}$ & OD & $\mathrm{OE}$ \\
\hline $1^{*}$ & $20 / 80$ & $20 / 100$ & J4 & J5 \\
\hline $2^{*}$ & $20 / 50$ & $20 / 80$ & $\mathrm{~J} 4$ & $\mathrm{~J} 4$ \\
\hline $3^{*}$ & $20 / 400$ & $20 / 100$ & J6 & J4 \\
\hline 4 & $20 / 400$ & $20 / 100$ & J4 & J4 \\
\hline $5^{*}$ & CD 1 metro & $20 / 400$ & $\mathrm{~J} 4$ & $\mathrm{~J} 2$ \\
\hline $6^{*}$ & CD 1 metro & CD 0,5 metro & $<J 6$ & $<J 6$ \\
\hline $7^{*}$ & Não informa & Não informa & Não informa & Não informa \\
\hline 8 & $20 / 150$ & $20 / 400$ & $\mathrm{~J} 6$ & J6 \\
\hline $9^{*}$ & $20 / 80$ & $20 / 100$ & $\mathrm{~J} 2$ & $\mathrm{~J} 1$ \\
\hline 10 & $20 / 400$ & $20 / 400$ & $\mathrm{~J} 6$ & J6 \\
\hline 11 & $20 / 70$ & $20 / 70$ & $\mathrm{~J} 2$ & J3 \\
\hline $12^{*}$ & Sem percepção luminosa & Percepção luminosa & $<\mathrm{J} 6$ & $<\mathrm{J} 6$ \\
\hline $13^{*}$ & CF 1 metro & $20 / 150$ & $<J 6$ & J6 \\
\hline 14 & $20 / 80$ & $20 / 50$ & J3 & J3 \\
\hline $15^{\star}$ & $20 / 80$ & $20 / 80$ & J4 & $\mathrm{J} 4$ \\
\hline 16 & $20 / 100$ & $20 / 100$ & $<\mathrm{J} 6$ & $<\mathrm{J} 6$ \\
\hline 17 & $20 / 100$ & $20 / 100$ & $<J 6$ & $\mathrm{~J} 6$ \\
\hline $18^{*}$ & Percepção luminosa & Sem percepção luminosa & $<\mathrm{J} 6$ & $<\mathrm{J} 6$ \\
\hline 19 & $20 / 80$ & $20 / 80$ & J3 & J4 \\
\hline $20^{*}$ & $20 / 200$ & $20 / 400$ & $\mathrm{~J} 4$ & J3 \\
\hline
\end{tabular}

cinco com astigmatismo miópico. Cinco idosos (25\%) apresentaram hipermetropia, destes 10 olhos cinco eram afácicos após cirurgia de catarata, sendo 2 idosos com afacia binocular e 1 idoso com um olho afácico e o outro pseudofácico. Miopia foi encontrada em 4 idosos em ambos os olhos (20\%). Um idoso era amaurótico de um olho e tinha percepção luminosa no olho adelfo por glaucoma, sem melhora da AV independentemente do tipo de correção usada.

Novos óculos foram prescritos para 9 idosos (45\%) e melhora na acuidade visual foi obtida em 4 casos (20\%). A melhora da acuidade visual ocorreu em 3 casos de 20/200 para 20/100 e 1 caso de 20/400 para 20/200.

A citologia mostrou, em todos os idosos, a presença de células epiteliais em degeneração e a cultura de cílios e conjuntiva: Staphylococcus coagulase negativa e Corynebacterium xerosis.

Em relação à avaliação da lágrima, 12 idosos (60\%) apresentaram tempo de ruptura do filme lacrimal alterado, menor que 10 segundos, dentre os quais cinco também apresentaram teste de Schirmer basal alterado, não atingindo $15 \mathrm{~mm}$ na fita em cinco minutos.

Pressão intra-ocular, acima de $20 \mathrm{mmHg}$ foi encontrada em três idosos (15\%) acompanhada de aspecto sugestivo de lesão glaucomatosa do nervo óptico. Somente nestes casos a escavação estava alterada: um com escavação total bilateral, o outro com escavação de 0,7 em um dos olhos e 0,8 no olho adelfo e o último com escavação de $0,4 \mathrm{em}$ um olho e total no outro acompanhada de atrofia. Nenhum dos idosos estava em uso de hipotensor ocular.

Ao exame de biomicroscopia, vinte e quatro olhos (60\%) apresentaram algum tipo de opacidade do cristalino. Dezesseis $(40 \%)$ haviam sido submetidos à cirurgia de catarata. Pseudofácicos com lente intra-ocular de câmara posterior 10 $(62,5 \%)$ e afácicos $6(37,5 \%)$.

Blefarite moderada a intensa foi observada em 4 idosos. Afinamento conjuntival possibilitando a observação dos músculos retos foi observada em três idosos.

Ao exame fundoscópico, rarefação e atrofia do epitélio pigmentar da retina (EPR) foi descrita em todos os idosos; assim como um afinamento generalizado do calibre vascular. A presença de degeneração macular relacionada à idade (DMRI), na forma seca, drusas ou sem cicatriz disciforme só drusas sem cicatriz disciforme ou atrofia moderada, foi encontrada em 14 idosos (70\%), 5 idosos (25\%) com DMRI moderada e severa e a forma exsudativa, já cicatrizada, foi descrita em um idoso em ambos os olhos. Aspecto miópico da retina com atrofia generalizada do EPR e áreas de degeneração miópica periféricas foi descrita em 4 idosos (20\%).

\section{DISCUSSÃO}

A predominância de mulheres no presente estudo está de acordo com o encontrado e descrito no senso demográfico. As mulheres tendem a viver mais que os homens ${ }^{(1,3)}$ e deve aumentar ainda mais com o passar dos anos; dado importante ao se pensar em um maior número de mulheres viúvas e vivendo sós com o aumento da longevidade. Caracterizar essa população idosa como predominantemente feminina ajuda de forma direta no planejamento de políticas de prevenção ${ }^{(3)}$. 
Colaborando para conhecer melhor esta população também está o fato de que doenças sistêmicas crônicas como a diabetes, o câncer e até as cardiopatias descritas como predominantes na terceira idade foram pouco encontradas na nossa amostra ${ }^{(3-4)}$. Cerca de $25 \%$ da amostra era sadia sem queixas e $40 \%$ dela apresentava algum tipo de tratamento anti-hipertensivo.

Em relação à queixa visual $40 \%$ referiram estar satisfeito com a sua atual visão e outros $55 \%$ reclamaram de insatisfação principalmente em relação à visão para perto. Esses dados estão de acordo com um estudo publicado em 2003 no qual o principal motivo de procura para atendimento oftalmológico era o desejo de retorno à leitura ${ }^{(11)}$. A importância dada à acuidade visual para perto deve ser registrada como meta importante nas políticas de prevenção, pois os idosos valorizam esta atividade ${ }^{(8)}$. Por outro lado, se pensarmos que à medida que os anos passam as atividades diárias tornam-se mais restritas e a necessidade da acuidade visual para atividades torna-se também menor, a exigência com relação à visão também diminui o que pode ajudar a explicar o fato dos $40 \%$ de idosos que referiram estar satisfeitos com a sua atual visão, apesar de baixas.

Os resultados encontrados com relação aos erros refrativos mais comuns estão de acordo com os encontrados por Rodrigues et al., uma predominância de astigmatismo. A presença de hipermetropia por afacia também se justifica se considerarmos que muitos desses indivíduos foram submetidos à cirurgia há 20 a $30 \operatorname{anos}^{(12)}$. Quanto à prescrição de óculos e a pouca melhora na acuidade visual promovida por estes pode ser justificada pelo fato da maioria destes idosos terem suporte e atenção familiar significativo, estando, portanto regularmente assessorados e com exame oftalmológico atualizado.

A diminuição do tempo de ruptura do filme lacrimal e teste de Schirmer basal alterados podem ser em decorrência da diminuição dos componentes aquoso e mucínico da lágrima ${ }^{(13)}$.

Os achados de catarata como principal fator de interferência na acuidade visual, seguida pela DMRI, concordam com outros estudos $^{(12)}$. Já a incidência de glaucoma na nossa população foi de $5 \%$ o que difere dos dados da literatura. Os dados encontrados na nossa amostra também coincidem, em parte, com outros estudos realizados no tocante às principais afeccções oculares diagnosticadas: ametropias, cataratas e glaucoma $^{(12,14-15)}$. Nos mesmos estudos as principais causas de deficiência visual, também coincidem, foram catarata e degeneração macular relacionada à idade ${ }^{(12,14-15)}$.

A importância de políticas que permitam acesso à cirurgia de catarata a população como um todo passa a ter enorme significado e potencial de melhoria na qualidade de vida destas pessoas, assim como, investir em pesquisa para avanços no tratamento da DMRI.

Odom reafirma que a visão interfere na manutenção da saúde de um indivíduo e que a sua melhora muitas vezes pode ser atingida se a devida atenção for dada a esta população(14). Bergman em seus estudos em população de 70 a 97 anos defende que para maioria das pessoas idosas a deterioração na acuidade visual não quer dizer necessariamente uma mu- dança negativa na habilidade visual ${ }^{(1,7)}$. Por outro lado, Manino demonstrou que as pessoas com idade entre os 65 e 74 já tem pelo menos três a quatro vezes maior dificuldade em andar, sair de casa e se deitar ${ }^{(16)}$. Mesmo que existam divergências, as colocações acima vêm reforçar a importância que um trabalho conjunto de prevenção e estudos para tratamento específico desta nova população idosa só pode trazer vantagens na busca de novas perspectivas.

Embora a nossa amostra seja pequena em número impedindo generalizar os achados descritos, permite, assim mesmo, chamar a atenção para pontos relevantes. Importante ressaltar que trabalhos com populações acima dos 90 anos foram pouco encontrados na literatura, tornando difícil comparar diretamente os dados aqui encontrados embora por outro lado valorize a pesquisa realizada.

Concluindo, determinar as alterações visuais presentes em indivíduos com mais de 99 anos colabora com informações para traçar novas diretrizes, políticas de prevenção e tratamento permitindo uma melhor qualidade de vida para essa nova população de idosos.

\section{ABSTRACT}

Purpose: To determine vision conditions and ocular findings in patients older than 99 years. Methods: Patients were recruited by media advertisement and examined at the Vision Institute of UNIFESP. The following examinations were performed: visual acuity, ectoscopy, refraction, biomicroscopy, tear film break-up time, Schirmer basal test, tonometry, direct and indirect ophthalmoscopy. Complementary examinations such as optical coherence tomography were performed when indicated. Results: Thirty patients older than 99 years were identified. Mean age of 101.5 $\pm 1.8,25$ females and 5 males. Most common systemic disease, according to history, was arterial hypertension. In 11 patients $(55 \%)$, the best corrected far visual acuity was $20 / 100$ or better. The best corrected near visual acuity was $\mathrm{J} 4$ or better also in 55\% (11 patients) and $63.0 \%$ (7 patients) of these were the ones that had not been submitted to cataract surgery. The most frequent visual complaint was low vision for reading $(55 \%)$ and other 8 patients $(40 \%)$ reported no disturbance (satisfied with their vision). Lens opacity was present in 9 patients $(60 \%)$ and 8 patients (40\%) had previous cataract surgery ( 5 aphakic eyes, 9 pseudophakic eyes). Cataract was identified as important for impairment of vision in 5 patients but only 2 of them wanted to be submitted to surgery. The main cause of visual impairment was age-related macular degeneration, present in all patients, $95 \%$ with no exudative form. Vision improved with new refractive prescription in 4 patients. Conclusion: Once we understand the needs we can plan specific diagnostic and treatment strategies. This sample showed that most were female $(83.3 \%)$, with good general health (35\%), most with systemic arterial hypertension $(40 \%)$, complaining of low vision for reading $(55 \%)$ because of age-related macular degeneration $(100 \%$, 
95\% dry form). Only $40 \%$ had been previously submitted to cataract surgery. Most of the patients with cataract said to be satisfied with their vision; of the 5 patients that could be operated only 2 decided to do it. Low vision for reading, AMD and cataract were the main findings.

Keywords: Demographic aging; Aging/physiology; Vision disorders; Aged, 80 and over; Health services for the aged; Visual acuity; Quality of life

\section{REFERÊNCIAS}

1. Bergman B, Sjostrand J. A longitudinal study of visual acuity and visual rehabilitation needs in an urban Swedish population followed from the ages of 70 to 97 years of age. Acta Ophthalmol Scand. 2002;80(6):598-607.

2. West S, Sommer A. Prevention of blindness and priorities for the future. Bull World Health Organ. 2001;79(3):244-8. Comment in: Bull World Health Organ. 2002;80(5):419.

3. Veras R. Em busca de uma assistência adequada à saúde do idoso: revisão da literatura e aplicação de um instrumento de detecção precoce e de previsibilidade de agravos. Cad Saúde Pública. 2003;19(3):705-15.

4. Camargo EF, Toledo MA, Guimarães RM. O velho e o médico: a anamnese diferenciada. Brasília Méd. 2001;38(1/4):42-6.
5. Xavier FM, Ferraz MP, Marc N, Escosteguy NU, Moriguchi EH. Elderly people's definition of quality of life. Rev Bras Psiquiatr. 2003;25(1):31-9.

6. Marx MS, Werner P, Cohen-Mansfield J, Feldman R. The relationship between low vision and performance of activities of daily living in nursing home residents. J Am Geriatr Soc. 1992;40(10):1018-20.

7. Bergman B, Bergstrom A, Sjostrand J. Longitudinal changes in visual acuity and visual ability in a cohort followed from the age of 70 to 88 years. Acta Ophthalmol Scand. 1999;77(3):286-92.

8. Kalache A, Veras RP, Ramos LR. O envelhecimento da população mundial: um desafio novo. Rev Saúde Pública. 1987;21(3):200-10.

9. Carvalho JAM, Garcia RA. O envelhecimento da população brasileira: um enfoque demográfico. Cad Saúde Pública. 2003;19(3):725-34.

10. Pereira SR, Curion CC, Veras R. Perfil demográfico da população idosa no Brasil e no Rio de Janeiro em 2002. Text Envelhec. 2003;6(1):43-59.

11. Lucas MB, Leal DB, Tavares SS, Diniz JR, Cantanhede MI. Baixa visão em idosos - estudo comparativo entre um serviço público e um serviço privado da Cidade do Recife. Rev Bras Oftalmol. 2003;62(2):138-43.

12. Rodrigues JLT, Celino AC, Diniz JR, Celino M, Florêncio T. Avaliação oftalmológica em idosos de Jabotão-PE. Rev Bras Ciênc Saúde. 2001;5(2):119-24.

13. Matayoshi S, Kikuta H. Vias lacrimais e senilidade. In: Kara Jose N, Almeida GV de, organizadores. Senilidade ocular. São Paulo: Roca; 2001. p.191-3.

14. Odom JV. Vision, visual needs, and quality of life of older people in rural environments: a report and synthesis of a meeting. J Rural Health. 2001;17(4): 360-3.

15. Medina NH. Epidemiologia do envelhecimento: estudo oftalmológico populacional de idosos. [tese]. São Paulo: Universidade Federal de São Paulo;1997.

16. Manino JH. Visual deficits and mobility. Evaluation and management. Clin Geriatr Med 1996;12(4):803-23. 